Case report

\title{
Successful treatment of Candida parapsilosis and Pseudomonas aeruginosa infection using medical and surgical management in an injecting drug user with mitral and aortic valve endocarditis: a case report Hanady Daas ${ }^{1}$, Fadi Abuhmaid ${ }^{1}$ and Marcus Zervos ${ }^{1,2,3 *}$
}

Address: ${ }^{1}$ Department of Internal Medicine, Henry Ford Hospital, 2799 W Grand Blvd, Detroit, MI 48202, USA, ${ }^{2}$ Department of Infectious Disease, Henry Ford Hospital, 2799 W Grand Blvd, Detroit, MI 48202, USA and ${ }^{3}$ Wayne State University, Detroit, MI, USA

Email: HD - Hdaas1@hfhs.org; FA - fabuhma1@hfhs.org; MZ* - Mzervos1@hfhs.org

* Corresponding author

\author{
Published: 8 May $2009 \quad$ Received: 8 January 2008 \\ Journal of Medical Case Reports 2009, 3:6598 doi: 10.1186/1752-1947-3-6598 Accepted: 22 January 2009 \\ This article is available from: http://jmedicalcasereports.com/jmedicalcasereports/article/view/6598 \\ (c) 2009 Daas et al; licensee Cases Network Ltd. \\ This is an Open Access article distributed under the terms of the Creative Commons Attribution License (http://creativecommons.org/licenses/by/3.0), \\ which permits unrestricted use, distribution, and reproduction in any medium, provided the original work is properly cited.
}

\begin{abstract}
Introduction: Polymicrobial endocarditis is a well-recognized problem in intravenous drug users and it accounts for I to $3 \%$ of endocarditis cases overall and up to $9 \%$ in other series. The most common combinations of organisms include Staphylococcus aureus and Streptococcus pneumoniae followed by Staphylococcus aureus and Pseudomonas aeruginosa. Candida parapsilosis endocarditis carries a mortality rate of $45 \%$, and each infection with Candida or Pseudomonas endocarditis per se carries a very high mortality rate approaching $85 \%$ and $80 \%$, respectively. The combination of $P$. aeruginosa and $C$. parapsilosis has never been encountered and there have been no earlier reports of the combination of $C$. parapsilosis and $P$. aeruginosa in adult intravenous drug users as a cause of endocarditis.
\end{abstract}

Case presentation: We present a 49-year-old man with bivalvular endocarditis with $P$. aeruginosa and C. parapsilosis. He had a prior bivalvular replacement in 2005 that became infected with the above microorganisms and he was treated with intravenous antibiotics. Because of ongoing intravenous drug use, a second valve replacement was denied. A few days later, the patient presented with septic shock secondary to $P$. aeruginosa and $C$. parapsilosis recurrent endocarditis. The infection was cured with a second bivalvular replacement and extended therapy with antibiotics and antifungals.

Conclusion: This is the first time a patient has presented with $P$. aeruginosa and $C$. parapsilosis endocarditis. Relapsing polymicrobial endocarditis can be cured with medical and surgical therapy.

\section{Introduction}

Few data exist on infective endocarditis in intravenous drug use (IVDU) patients. A new pattern of infective endocarditis in IVDU is emerging, characterized by more frequent left heart involvement, a severe clinical course, and a need for surgery in the active phase [1]. The standard of care has included perioperative antifungal, radical debridement of infected tissue, reconstruction using 
biological tissue when possible, and prolonged oral suppressive antifungal therapy. Medical treatment of fungal endocarditis on prosthetic valves can be successful in selected cases. Left-sided endocarditis compared to right, and polymicrobial compared to single organism, are well known risk factors for an increase in morbidity and mortality in intravenous drug users with infective endocarditis [2].

\section{Case presentation}

A 49-year-old man with a history of intravenous drug abuse, mainly heroin, presented to the emergency department in Henry Ford Hospital where he complained of chills and diarrhea. The diarrhea was watery and continuous of 1-day duration accompanied by chills. He denied shortness of breath or chest pain.

Our patient was hospitalized in another facility 14 days before presenting to the emergency department of our hospital, and was found to have Candida parapsilosis and Pseudomonas aeruginosa endocarditis. A 2D echocardiogram was done there and showed vegetation of the prosthetic mitral valve, with normal aortic prosthesis. He was discharged with a peripherally inserted catheter (PIC) line and on intravenous antibiotics namely, tobramycin, cefepime and fluconazole. No valve replacement was performed at that time due to ongoing injection drug use. He was only out for 1 day before presenting to our emergency department.

His past medical history was significant for aortic and mitral valve replacement 1 year before this hospitalization for endocarditis. He also had stage 3 chronic kidney disease.

At initial presentation, he was found to be febrile at $100.3^{\circ} \mathrm{F}$ and hypotensive at a blood pressure of $85 / 50$. His physical examination revealed a $3 / 6$ systolic murmur in the aortic area with radiation to the precordium, and a 2/6 diastolic murmur over the mitral area without radiation.

His initial laboratory investigations showed white blood cell count of $19.2 \mathrm{k} / \mu \mathrm{L}$ with $93 \%$ neutrophils, hemoglobin of $9.6 \mathrm{~g} / \mathrm{dL}$, blood urea nitrogen (BUN) $26 \mathrm{mg} / \mathrm{dL}$, creatinine $2.2 \mathrm{mg} / \mathrm{dL}$, serum bicarbonate $16 \mathrm{mmol} / \mathrm{L}$, chloride $112 \mathrm{mmol} / \mathrm{L}$, sodium $136 \mathrm{mmol} / \mathrm{L}$, magnesium $1.2 \mathrm{mmol} / \mathrm{L}$. Prothrombin time was 15 seconds, and international normalized ratio (INR) was 1.17. Urine toxicology screen was positive for benzodiazepines. Troponin level was $2.2 \mathrm{ng} / \mathrm{mL}$ (normal<0.4). Initial blood cultures were drawn in the emergency department and later grew $P$. aeruginosa susceptible to amikacin, aztreonam, cefepime, ciprofloxacin, imipenem, piperacillin/tazobactam and tobramycin. Serology was positive for Hepatitis C infection and negative for HIV infection.
On presentation, he was admitted to the intensive care unit with the diagnosis of septic shock. He was started on intravenous vancomycin, tobramycin, micafungin and cefepime.

Three sets of blood cultures grew C. parapsilosis susceptible to fluconazole, itraconazole and 5 flucytosine, and $P$. aeruginosa susceptible to amikacin, aztreonam, cefepime, ciprofloxacin, imipenem, piperacillin/tazobactam and tobramycin.

A 2D echocardiogram showed a bioprosthetic aortic valve with a medium, $10 \mathrm{~mm}$, pedunculated, highly mobile vegetation of the aortic valve attached to the posterior aortic annulus. There was no significant aortic valvular regurgitation. There was mild mitral valvular regurgitation.

At day 2 of hospitalization, a transesophageal echocardiogram was done. It showed no aortic abscess but small, $5 \mathrm{~mm}$, mobile vegetation of the aortic valve attached to the non-coronary cusp without significant regurgitation. It also showed large, $10 \mathrm{~mm}$, mobile mitral valve vegetation attached to the anterior leaflet without significant regurgitation.

The hospital course was complicated by acute renal failure and acute heart failure for which the patient was aggressively resuscitated. He was electively intubated and medically stabilized to undergo valve replacement 5 days later. Ethical and logistic considerations were the focus of an extensive discussion among the different treating medical and surgical teams given the active injecting drug use while being treated for a relapse of infective endocarditis. The decision was finally made to proceed with the valve replacement surgery. The patient was kept in hospital for the duration of intravenous antibiotics to ensure adherence to therapy and to prevent use of the intravenous access for illicit drug use.

At day 7 of hospitalization, he underwent aortic and mitral valve replacement. Cultures from the valves also grew P. aeruginosa and C. parapsilosis. All blood cultures drawn after day 1 were negative.

Tobramycin and cefepime were given for 6 weeks postoperatively. Tobramycin trough levels were measured frequently to ensure therapeutic levels. Micafungin was given for 5 days postoperatively, and replaced by anidulafungin that was given for 26 days. Fluconazole was given for 30 days postoperatively while in hospital and prescribed for 3 months after discharge from the hospital.

Throughout hospitalization, he remained afebrile and euvolemic without symptoms of heart failure. Two weeks 
after discharge from the hospital, he returned to the infectious disease clinic, being in good health, without history of fever, excess sweating, chest pain or shortness of breath. Follow-up blood cultures were drawn and were negative.

\section{Discussion}

Polymicrobial endocarditis is a growing problem and has been attracting increasing attention over the past decade $[3,4]$. The etiology in IVDU is changing, comprising Staphylococcus, Pseudomonas and other pathogenic fungi $[5,6]$. Most commonly isolated fungi are C. albicans (24\%). Non albicans candida accounts for another $24 \%$ of the fungal isolates $[6,7]$. The difficulty arises in the management of these cases, especially with the growing resistance to the usual combination antibiotics [8].

This is the first time a patient has presented with $P$. aeruginosa and C. parapsilosis endocarditis. Optimal treatment options and cure rate remain to be evaluated. We present a man who had successful treatment with a combination of antibiotics and antifungals in addition to surgical treatment.

\section{Conclusion}

Polymicrobial endocarditis with C. parapsilosis and $P$. aeruginosa in intravenous drug users can be cured with surgical valve replacement and extended duration intravenous antibiotics and antifungals.

\section{Abbreviations}

BUN, blood urea nitrogen; IDU, injecting drug user; INR, international normalized ratio; IVDU, intravenous drug use; PIC, peripherally inserted catheter.

\section{Consent}

Written informed consent was obtained from the patient for publication of this case report and any accompanying images. A copy of the written consent is available for review by the Editor-in-Chief of this journal.

\section{Competing interests}

The authors declare that they have no competing interests.

\section{Authors' contributions}

HD initiated the case report, obtained the consent, and gathered the patient's data. FA reviewed the article, provided some data and contributed to the discussion. $\mathrm{MZ}$ supervised and edited the case report and provided some of the references.

\section{References}

I. Mathew J, Addai T, Anand A, Morrobel A, Maheshwari P, Freels S: Clinical features, site of involvement, bacteriologic findings, and outcome of infective endocarditis in intravenous drug users. Arch Intern Med 1995, I55(15): I64 I-I648.
2. Carozza A, De Santo LS, Romano G, Della Corte A, Ursomando F, Scardone $M$, Caianiello $G$, Cotrufo $M$ : Infective endocarditis in intravenous drug abusers: patterns of presentation and long-term outcomes of surgical treatment. J Heart Valve Dis 2006, I5(I):|25-|3|.

3. Hoen B, Alla F, Selton-Suty C, Béguinot I, Bouvet A, Briançon S, Casalta JP, Danchin N, Delahaye F, Etienne J, Le Moing V, Leport C, Mainardi JL, Ruimy R, Vandenesch F: Association pour I'Etude et la Prévention de I'Endocardite Infectieuse (AEPEI) Study Group: Changing profile of infective endocarditis: results of a I-year survey in France. JAMA 2002, 288:75-8I.

4. Brito LR, Guimarães T, Nucci M, Rosas RC, Paula Almeida L, Da Matta DA, Colombo AL: Clinical and microbiological aspects of candidemia due to Candida parapsilosis in Brazilian tertiary care hospitals. Med Mycol 2006, 44(3):26I-266.

5. Shively BK: Infective endocarditis. In Current Diagnosis \& Treatment in Cardiology. 2nd edition. Edited by Crawford MH. McGraw Hill; 2003.

6. Ellis ME, Al Abdly A, Greer W, Ventura W: Fungal endocarditis: evidence in the world literature 1965-1995. Clin Infect Dis 200I, 32(I):50-62.

7. Butkevich OM, Vinogradova TL: [Some problems of the current therapy of infective endocarditis.] Ter Arkh 2000, 72(9):54-57.

8. Moreillon P, Que Y: Infective endocarditis. Lancet 2004, 363:|39-|49.

\section{Do you have a case to share?}

Submit your case report today

- Rapid peer review

- Fast publication

- PubMed indexing

- Inclusion in Cases Database

\section{Any patient, any case, can teach us something}

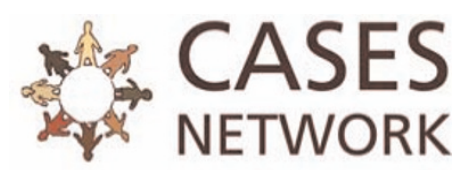

www.casesnetwork.com 